\title{
Die Rolle des Staates - zum Beispiel im Gesundheitswesen
}

M. Notter

Der Sozialstaat soll vollkommen sein. Dieser Anspruch gilt auch für den Gesundheitsbereich. Hier stehen sich der Einsatz zur Krankenheilung und Lebensrettung und die planerische Administration gegenüber. Während jedoch die Erwartungen an die Länge des menschlichen Lebens stetig steigen, bleibt die Zeit für beide Aufgaben endlich. Je mehr Administration, um so weniger Krankenheilung und Lebensrettung. Dazu gibt es im Gesundheitswesen immer wieder eingebaute Multiplikatoren für die Ausgaben, aber keine Begrenzungen. Die heutige Situation vermag die Politik nicht einfach zu lösen. Denn weder der Wunsch nach Ausbau des Systems noch der Wille zum Sparen sind mehrheitsfähig. Wenn man am Status quo etwas ändern will, dann braucht das Information und Kommunikation, Markt und damit Wettbewerb sowie Transparenz und Fokus.

\section{Ausgangslage}

Wer seinen Beruf erlernt hat, um Kranke zu heilen und Leben zu retten, ist selten ein Freund der Administration. Denn sein Leben verläuft von Notfall zu Notfall, für beschauliches Nachdenken bleibt im Wettlauf mit der Stundenuhr kaum Zeit. Demgegenüber verfügt der Planer über die Illusion des klärenden Abstands; er und ihm hörige Bürger erliegen einer Versuchung mit dem Ruf nach Koordination und Abstraktion: Es entstehen mindestens zwei Welten, die sich mit hohen ungleichen Erwartungen gegenüberstehen. Eine Brücke der Verständigung zwischen diesen beiden Welten erscheint um so unmöglicher, als der Arzt auszurechnen beginnt, wieviel ihn die Administration von der eigentlichen Aufgabe der Krankenheilung und Lebensrettung abhält, während der Planer seinen Beitrag als hilfreich im Interesse der nach Harmonie strebenden umverteilenden Gesellschaft in ihrem Wahn nach staatlicher Vollkommenheit versteht. Die Frage wird in einem Umfeld des Neids gestellt, wo der Durchschnittsbürger nicht nachvollziehen kann, dass Befähigung zur Heilung und Lebensrettung seit Jahrhunderten grosses (Fach-)Wissen und Erfahrung voraussetzen muss (mit Ausbildungsfolgen und dem Anspruch auf eine Null-Fehler-Kultur), um so mehr als die Folgen eines Fehlers irreversibel sind, Leid zufügen und von der Gesellschaft geächtet werden.

Das Individuum, das ja Bestandteil der Gesellschaft ist, stellt in seinem Streben nach dem glückseligen ewigen irdischen Leben Ansprüche nach Vollkommenheit an den (Sozial-)Staat. Dieser soll die Existenz sichern, zu der auch mindestens seit der Einführung des Obligatoriums der Krankenversicherung - die Gesundheit gehört.

Als Retter in der Not - zwecks Sicherstellung des Traums vom ewigen Leben - werden Leistungsfinanzierer (Krankenkassen) eingesetzt, die an einen Grundkatalog von Leistungen gebunden sind, der durch die Politik bestimmt wird, die es «recht machen will». Nur so ist der Einbezug von Gewichtabsaugen, Geschlechtsumwandlung und Komplementärmedizin - um Beispiele zu nennen - in den Grundkatalog zu verstehen.

Träume platzen, denn es gilt der Satz auch hier, dass «die «Erfolge» von gestern die Herausforderungen von morgen» sind. Das gilt, wie zu zeigen sein wird, auch für das Gesundheitswesen und sein Verhältnis zum Bürger und für den Staat und dessen Rolle in der Gesellschaft.

\section{Zur Rolle des Staates}

Der Sozialstaat hat mittlerweile so viele Fragen aufgeworfen, wie er vorgibt zu lösen. Einige davon, die heute miteinander verknüpft sind, verlangen nach einer neuen Antwort; sie sind eine Herausforderung. Der Bürger spürt das zuerst in seinem Geldbeutel, die gegenwärtig starke Vorbelastung der Bruttolöhne durch Gemeinschaftsaufgaben, erinnert an die Zeiten des Naturallohnes, ein System, das durch die Geldwirtschaft abgelöst wurde. Weil mehr als die Hälfte der erwirtschafteten Substanz durch den Staat vordisponiert wird, besteht die Gefahr, dass in die Schwarzarbeit ausgewichen wird, wo nichts abgezogen wird und die Geldwirtschaft wieder funktioniert, wie das Beispiel Deutschland eindrücklich zeigt. Denn nun wissen wir es alle: Der Sozialstaat ist teuer, und er besteht aus Massnahmen der Umverteilung, was auch zu wach- 
senden Ausgaben führt. Sicher handelt es sich bei den Elementen des Sozialstaates nicht um einmalige Reparaturen, schon gar nicht im Bereich des Gesundheitswesens, denn man kann immer noch mehr tun, und man kann dies als Politiker auch gut begründen. Für viele wird die medizinische Versorgung zu einer schier unbegrenzbaren Verpflichtung. Was Wunder, dass es im Gesundheitswesen immer wieder eingebaute Multiplikatoren für die Ausgaben, aber keine Begrenzungen gibt. Dies führt dazu, dass wir erkennen müssen, dass es im Prinzip unmöglich ist, immer teurere Leistungen für alle zu finanzieren. Das erkennt und vor allem sagt niemand gerne.

Sie wissen, dass die Fragen, zu deren Lösung der traditionelle Sozialstaat gebaut wurde, zum einen in der Zeit des wirtschaftlichen Wachstums und der Hochkonjunktur entwickelt wurden und dass er zum andern gar nicht mehr die Fragen auf die Herausforderungen von morgen beantworten kann. Sie erinnern sich, der Sozialstaat wurde auf der Annahme der Arbeitsgesellschaft aufgebaut; er wird von Beschäftigten finanziert und ist auf Menschen ausgerichtet, die in der Berufswelt zu Hause sind. Nicht nur der Blick auf die tatsächliche Arbeitssituation in unserem Land, auch die Erkenntnisse der Demographie verdeutlichen rasch, dass die Grundlage des Sozialstaates in der Schweiz nicht mehr besteht, denn die Arbeitsgesellschaft ist nur noch ein Teil der Realität. Die Kategorien der Arbeitsgesellschaft reichen derzeit nicht mehr aus, um vorherzusagen, was die Menschen wünschen und wollen. Verstehen auch Sie die «Armen» als feste Schicht, die im Elend verharrt und durch die Wirtschaft in diese Lage versetzt wurde, wo doch nebst Arbeitslosigkeit heute vor allem Zivilstandsverhalten die Armutsfaktoren bildet? Die Frage ist erlaubt, ob Sozialversicherungssysteme im 21. Jahrhundert mit Grundkatalog, Lohnfortzahlungen, Arbeitslosengeldern an die völlig Mittellosen angeknüpft werden müssen oder ob man nicht zugeben will, dass wir es mit einer wohlhabenden Gesellschaft zu tun haben, die Hilfe den wirklich Notleidenden zukommen lässt.

Gelegentlich ist zu vernehmen, die Lösung sei so komplex, weil wir in einem föderalen Staat leben. Richtig ist, dass die Kantone ein unverzichtbar wesentlicher Bestandteil der Schweizerischen Eidgenossenschaft sind. Richtig ist auch, dass der Föderalismus nebst einer Wirkung einen Preis hat. Die Alternative wäre ein Zentralstaat, dessen Administration noch extensiver funktionierte. Offenbar werden die Frage und die damit verbundenen möglichen Lösungen in der Tat als komplex beurteilt; wie sonst ist zu erklären, dass der Bereich Gesundheitswesen aus der NFA Neu- gestaltung des Finanzausgleichs und Neuverteilung der Aufgaben zwischen Bund und den Kantonen ausgeklammert wurde?

\section{Von der Rolle der Politik und der Rolle des Souveräns - eine Frage der Mehrheit}

Noch immer gibt es welche, die uns mehr von der gleichen (Umverteilungs-)Medizin verschreiben wollen: Sie gefährden unsere Freiheit. Andere bezweifeln den Weg der Umverteilung und wollen diesen rückgängig machen; sie könnten Rechte des Bürgers in Gefahr bringen. Die Dritten glauben dass mit ein paar Reparaturmassnahmen das System weiter nutzbar ist; sie lösen die Probleme nicht, sie vertagen sie und verhindern die Lösung, weil sie in die Vergangenheit blicken.

Hand aufs Herz: Wüssten Sie, was das Volk will, wenn zirka 50 Prozent für einen Ausbau des Systems sind und zirka 50 Prozent fürs Sparen, aber ohne Hinweis, wo genau, wie gespart werden soll - schon gar nicht beim Katalog der Grundleistungen oder dort, wo es einen persönlich betrifft? Wüssten Sie, was der Souverän will bzw. was für Prioritäten er bei der Erfüllung der Staatsaufgaben im allgemeinen will, wenn er nicht sagt, was für Politikfelder wie wichtig sind und was das kosten darf in Funktion der eingehenden Steuergelder? Der Politiker kann daraus einzig ablesen, dass keine Meinung mehrheitsfähig ist. Fazit: Er setzt sich zwar für etwas ein, das aber niemandem weh tut - er werkelt am bestehenden System herum und lässt sich von einzelnen Betroffenen gar noch attestieren, das sei gar nicht so schlecht. Fazit: Weil der Bürger nicht weiss, was er will, werden die Aufträge an die Politik unklar erteilt, der Leistungsfinanzierer wird teurer, die Politik stellt die falschen Weichen - die Fahne in den Wind, was man ihr genaugenommen in dieser Situation gar nicht übelnehmen kann. Der Bürger fühlt sich im Stich gelassen: «Die da oben in Bern!» machen ES sowieso falsch. Zum einen, weil die Interessengruppen die «Falschen» in die Kommissionen schicken (so hören die Politiker auf die Falschen), zum andern, weil «die Politiker machen, was sie wollen» (weil vom Bürger nicht richtig informiert). Bemerkbar machen sich die Lobbys.

Der Bürger erwartet vom allmächtigen Staat, dass er ihm «Gesundheit garantiere», wo er doch zunächst für seine eigenen Bedürfnisse aufkommen soll. Geholfen werden soll demgegenüber denen, deren Not am grössten ist und die am wenigsten in der Lage sind, für sich selbst aufzukommen. 
Fazit: Jedermann ist frustriert, man klopft sich auf die Schultern, denn «denen hat man ES gesagt», passiert ist nichts oder jedenfalls das Falsche. Und dabei leben wir in einem Staat, wo viele Projekte und Studien im Hinblick auf eine Lösung finanziert und bearbeitet wurden und werden. Natürlich gibt es Patentrezepte nicht; wo es fehlt, wissen (fast) alle; Synergien werden erkannt; sicher werden sie zuwenig genutzt.

\section{Vom Umsetzungs- zum Informations- und Kommunikationsproblem}

Bekanntlich weiss jeder nur das, worüber er informiert wird bzw. was ihn interessiert bzw. was ihn gerade betrifft. Es fehlt die Gesamtschau. Fragt sich, wer in dieser Situation als Meinungsbildner auftritt: die Landesregierung, die politischen Parteien, die diversen Lobbys? Sie alle beteuern, dass sie vom mündigen Bürger ausgehen. Woher aber bezieht dieser Bürger die Information, oder noch wichtiger: Wo findet er Hilfe in der Beurteilung der Information als Grundlage für seinen Entscheid? Ist Gesundheitspolitik ein Spiel der Interessen und ihrer Vertreter geworden? Will der Bürger, dass der Staat überall dreinredet: von der Gentechnologie zur In-vitro-Befruchtung, die Liste lässt sich beliebig ergänzen. Oder gibt es Bereiche, in denen der Staat die Hände davon lassen soll? Statt eines (Gesundheits)-Markts mit Angebot, Nachfrage, Produkten, (die nicht nur vom Staat bereitgestellt werden), Unternehmen im Markt, die eine Nische gefunden haben (zum Beispiel die Spitäler) und einem Preis wird der «Markt» verzerrt, die Sicht vernebelt, denkt jeder nur an sich, wird das Vertrauen zerstört, haben Schlagworte leichtes Spiel, der Bürger wird manipuliert. Und so haben wir nebst dem Umsetzungsproblem auch noch ein Informations- und ein Kommunikationsproblem.

\section{Wie geht es weiter?}

Wenn man am Status quo etwas ändern will, dann braucht das Information und Kommunikation, Markt und damit Wettbewerb sowie Transparenz und Fokus. Anstatt Einzelvorschlägen sind Strategien zu entwickeln: Wir alle müssen an der Lösung mitarbeiten. Das können wir beispielsweise verwirklichen (die Liste ist nicht abgeschlossen):

- Wenn der Bürger Informationen zu gewichten weiss und mündig handelt, weiss er, was er unter «weniger Staat» versteht, und erteilt der Politik klare, mehrheitsfähige Aufträge. Er nimmt als Kunde am Gesundheitsmarkt teil ...

- Das Berufsbild des Arztes wird ändern. Der Auftrag, Kranke zu heilen und Leben zu retten, wird in Funktion des Marktes präzisiert: Der Arzt wird zum Unternehmer und Anbieter von Leistungen im Gesundheitsmarkt.

- Spitäler beispielsweise werden zu Dienstleistungsunternehmen mit Leistungsaufträgen in einem möglichst wenig regulierten Markt.

- Produkte haben transparente Preise.

- Weil Information immer weniger als Holschuld verstanden wird, erwartet der Bürger, dass ihm die Information frei Haus geliefert wird. Dadurch fällt die Auseinandersetzung mit Gleich- und Andersdenkenden weg, allfällige Synergien gehen verloren, wichtige Inputs werden vergessen, Kontakte zur Verständigung werden nicht geknüpft, wo einige Stunden genügen würden.

Packen wir es an! 


\section{Herbstseminar der Liberalen Aktion}

\section{Thema}

Rolle des Staates zum Beispiel im Gesundheitswesen

\section{Programm}

10.15 Uhr:

Begrüssung / Einführung in das Seminarthema, Dr. M. Notter

10.25-11.10 Uhr: Was ist gesund? Gedanken über Menschen, Staaten und deren Wohlbefinden. Roger de Weck, Publizist, Zürich und Berlin, Mitglied des PENClubs und Stiftungsrat des Karlspreises in Aachen

11.15-11.55 Uhr: Den Staat neu definieren? Soziopolitische Betrachtungen. Professor Dr. Franz Jaeger, Ordinarius für Wirtschaftspolitik und Geschäftsleitender Direktor des Forschungsinstituts für Empirische Ökonomie und Wirtschaftspolitik an der Universität St. Gallen

12.00-12.40 Uhr: Wie könnte es wirklich weitergehen? Etappen einer Rekonvaleszenz. Beiträge von Persönlichkeiten aus verschiedenen Erfahrungsbereichen

12.45-13.45 Uhr: Mittagspause und Erfrischungen

13.45-14.40 Uhr: Podiumsdiskussion mit Publikumsbeteiligung

14.45 Uhr: Verabschiedung

\section{Datum}

Samstag, 23. Oktober 2004, 10.15-14.45 Uhr

Ort

Congress Center Seedamm Plaza, Pfäffikon SZ

\section{Tagungskosten}

Fr. 125.-/Person, Studenten gegen Präsentation des Ausweises Fr. 50.- inkl. Dokumentation und Erfrischungen.

\section{Anmeldung}

Liberale Aktion, Tel. 013632240 oder Mail info@liberale-aktion.ch. Online-Anmeldung auf www. liberale-aktion.ch

Die Schweizerische Ärztezeitung verlost 10 Gratisteilnahmen! Berücksichtigt werden Anmeldungen, die bis zum 17. Oktober 2004 an die Adresse der Redaktion gelangen, via E-Mail: redaktion.saez@emh.ch oder per Fax 0614678556. 